\title{
Restless Space, a Consumable Interior
}

\section{NEREA FELIZ}

University of Texas at Austin
Populating the urban fabric of the host environment with myriad objects for sale, the street market produces a brief, exuberant and perishable system of interior spaces. While the market is taking place, the semiotics of the domestic unexpectedly disguise the city's streets. With a fluctuating number of vendors and an oscillating volume of merchandise, street markets defy prescribed architectural boundaries, raising dilemmas about flexibility and design control when using standard architectural components to provide permanence. Although nominally outdoors, what streets markets thrive on is a captivating kind of interiority, a mutable medium, characterized by cycles of change. Rather than following architectural typologies, the design of permanent market stalls might profitably turn its focus to models of interior occupation.

Architecture's spatial demarcation element par excellence is the wall. Walls traditionally delimit space by separating interior from exterior, and one room from another. Doors, by opening and closing, simultaneously form and dissolve a boundary. Embracing this dual performance of the door maximizes spatial flexibility. For a travelling market competition in Morocco, we proposed a field of doors, where doors, released from their conventional reliance on frames and walls, become an autonomous system. The outcome is 'Restless Space', a field of changing thresholds which blurs the traditional inside-outside dichotomy. This proposal renders a fully reversible spatial experience that is neither inside nor outside.
A grid of hinged doors, perforated as pegboards, shelves and retractable awnings provides the platform for multiple spatial configurations to take place. The combination of the intrinsic flexibility of these three elements, exponentially increases the capacity for spatial transformation to occur. The design can accommodate fluctuating demands, its layout can adapt to the necessities of autonomous vendors of various scales. In this way, small local business can coexist with bigger vendors, increasing the chances of the market's endurance.

The design can be easily disassembled and reassembled in any other location. The light structure and shallow foundations of the proposal would produce a minimal impact on the site. Rather than relying on high tech solutions requiring major maintenance or specialized technology, 'Restless Space' consists of a very simple and inexpensive system, easy to manufacture, build, sustain and repair by local trades. The proposal aims to minimize energy consumption, benefiting from Casablanca's extraordinarily mild climate. Full climatic comfort can be achieved solely through wind control and shading. The door rotation captures prevailing winds in the summer, generating a relieving breeze. In the winter, the doors rotate to form a series of wind shields. The operable awning system of each door helps to provide shade and to shelter from rain. Commerce has always been one of the most common means by which we experience public activity. Doors are charged with symbolic meaning. A field of doors opening to the street, both literally and symbolically, welcome and maximize the flow of humans and goods.

Acknowledgements: Tino Goo \& Helen Lee 


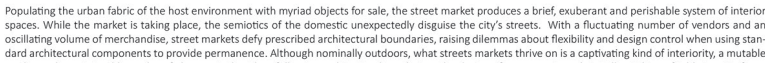

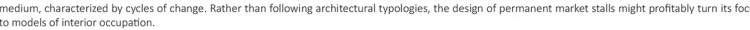

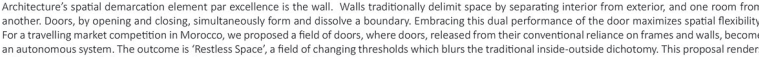
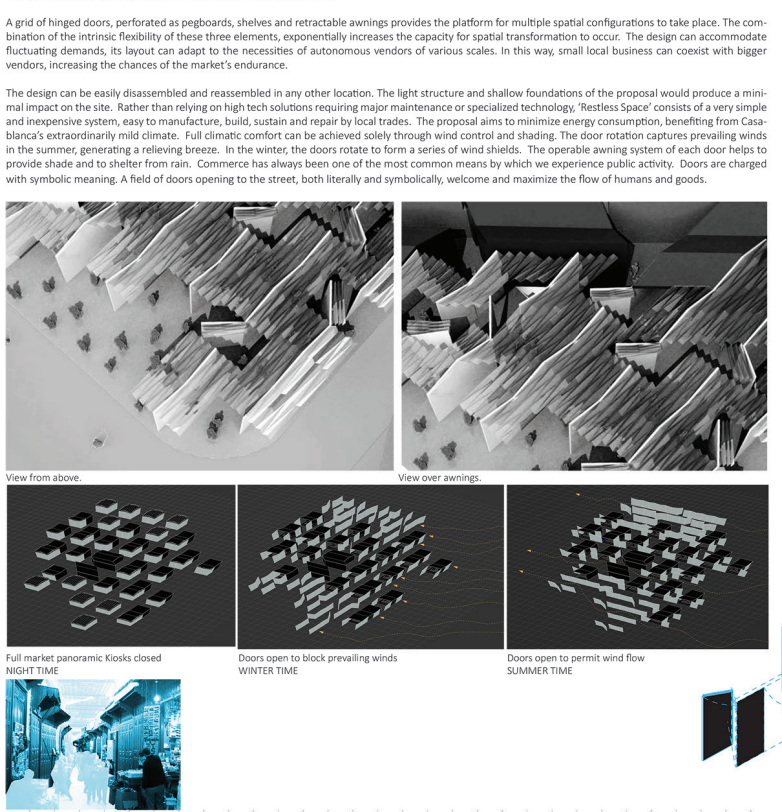

$=\mid \begin{array}{ll}1 \\ \frac{1}{3}\end{array}$
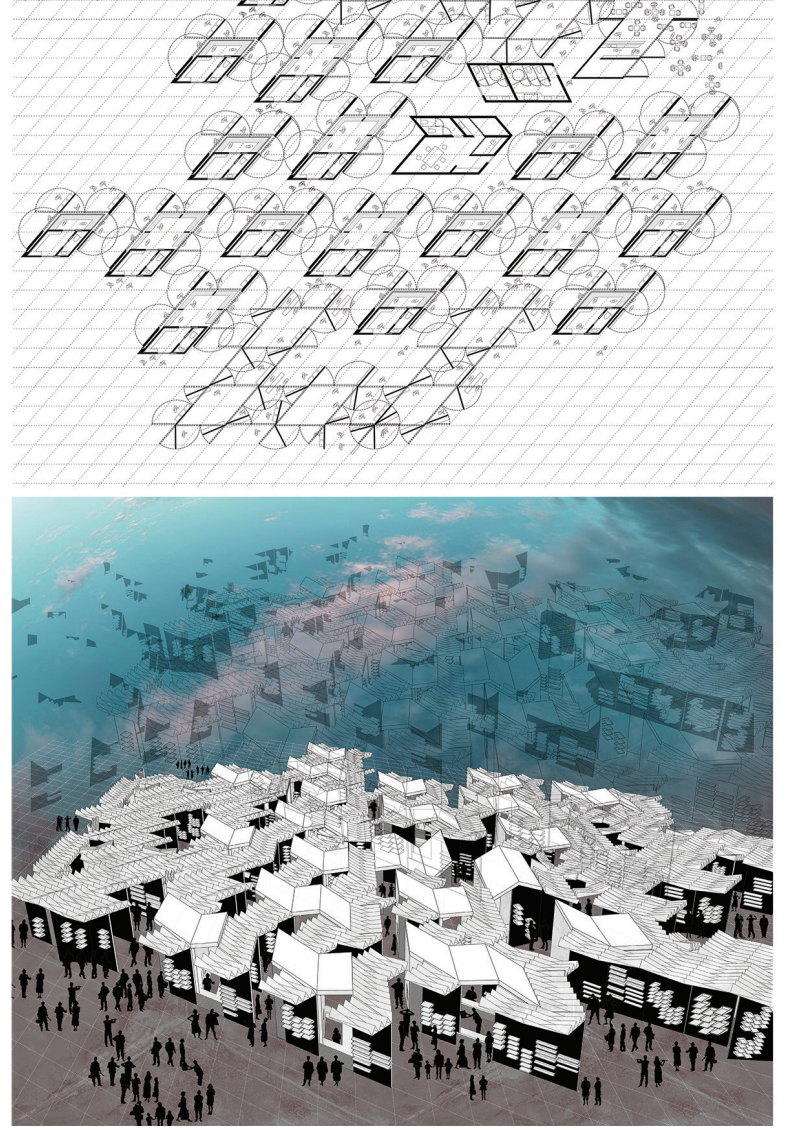

restless space A consumable Interior
for a Transitional Market in Morocco

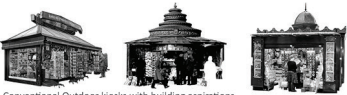
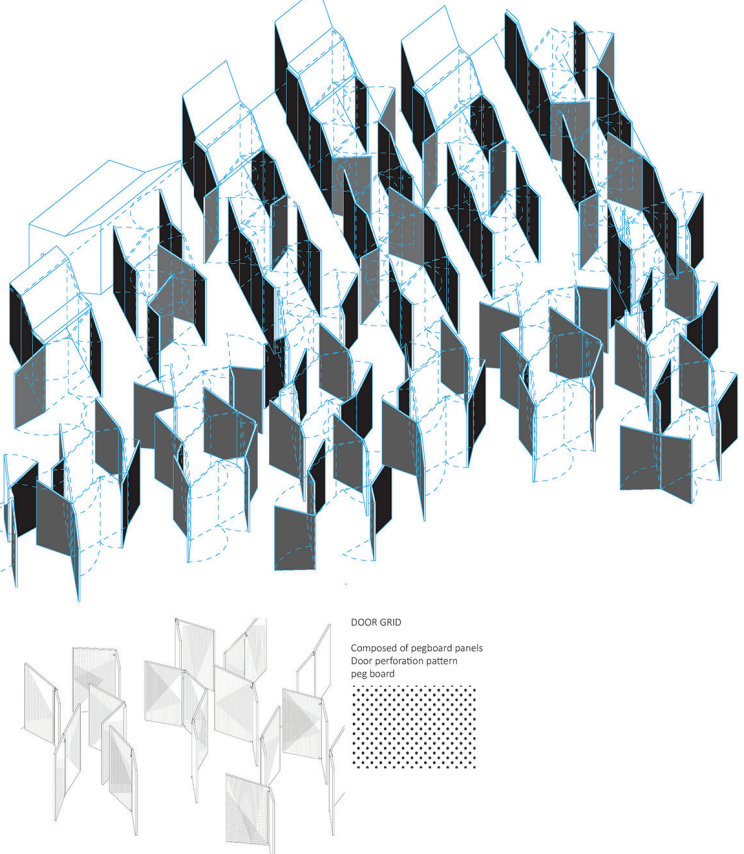

S

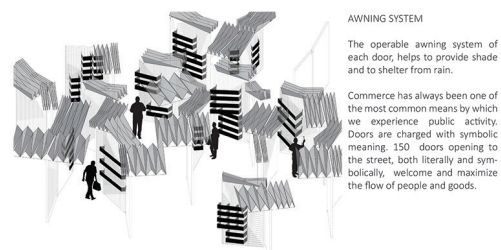

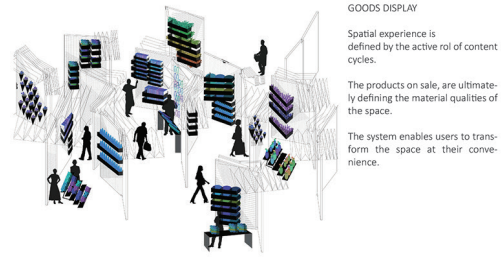

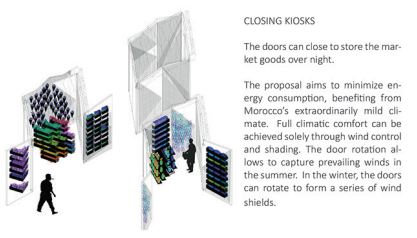

